Article

\title{
Wind and Wave Setup Contributions to Extreme Sea Levels at a Tropical High Island: A Stochastic Cyclone Simulation Study for Apia, Samoa
}

\author{
Ron Karl Hoeke *, Kathleen L. McInnes and Julian G. O’Grady \\ CSIRO Oceans and Atmosphere Flagship, PMB\#1 Aspendale, VIC 3195, Australia; \\ E-Mails: kathy.mcinnes@csiro.au (K.L.M.); julian.o’grady@csiro.au (J.G.O.) \\ * Author to whom correspondence should be addressed; E-Mail: ron.hoeke@csiro.au; \\ Tel.: +61-3-9239-4400 (ext. 123); Fax: +61-3-9239-4444.
}

Academic Editor: Rick Luettich

Received: 29 June 2015 / Accepted: 14 September 2015 / Published: 22 September 2015

\begin{abstract}
Wind-wave contributions to tropical cyclone (TC)-induced extreme sea levels are known to be significant in areas with narrow littoral zones, particularly at oceanic islands. Despite this, little information exists in many of these locations to assess the likelihood of inundation, the relative contribution of wind and wave setup to this inundation, and how it may change with sea level rise (SLR), particularly at scales relevant to coastal infrastructure. In this study, we explore TC-induced extreme sea levels at spatial scales on the order of tens of meters at Apia, the capitol of Samoa, a nation in the tropical South Pacific with typical high-island fringing reef morphology. Ensembles of stochastically generated TCs (based on historical information) are combined with numerical simulations of wind waves, storm-surge, and wave setup to develop high-resolution statistical information on extreme sea levels and local contributions of wind setup and wave setup. The results indicate that storm track and local morphological details lead to local differences in extreme sea levels on the order of $1 \mathrm{~m}$ at spatial scales of less than $1 \mathrm{~km}$. Wave setup is the overall largest contributor at most locations; however, wind setup may exceed wave setup in some sheltered bays. When an arbitrary SLR scenario $(+1 \mathrm{~m})$ is introduced, overall extreme sea levels are found to modestly decrease relative to SLR, but wave energy near the shoreline greatly increases, consistent with a number of other recent studies. These differences have implications for coastal adaptation strategies.
\end{abstract}


Keywords: storm surge; coral reefs; waves; sea level; islands; climate; tropical cyclone

\section{Introduction}

Tropical cyclones pose a significant hazard for many small island nations and have been estimated to account for $76 \%$ of natural disasters in the Pacific [1]. The generation of extreme coastal sea levels from storm surges and tides (referred to here as storm tides) and wind-waves are particularly hazardous for coastal communities, many of which are expanding through population pressure and tourism [2]. Understanding the likelihood of extreme sea levels under present and future climate conditions is, therefore, important for resilient coastal habitation and development. However, sea level observations, from which the likelihood of extreme sea levels can be assessed, are often of insufficient number and length to enable robust assessments, particularly given the relative infrequency of tropical cyclone (TC) occurrences at any given coastal location [3].

Extreme sea levels arise from a combination of factors including astronomical tides, storm surges, and wave breaking processes that lead to wave setup and run-up. Storm surge is caused by the inverse barometer effect (IBE) together with surface wind stress acting over coastal seas (which produces wind setup, see for example Pugh [4]). Wave setup is the increase in mean water levels due to the wind-wave dissipation and wave run-up is the maximum extent of the instantaneous wave uprush at the coast. These contributions have a morphological dependence: storm surge tends to be the dominant contributing factor on wide-shelved continental coastlines, and wave setup is often assumed to contribute less than 10\% to the total water level (e.g., [5,6]). Wave setup and run-up, on the other hand, have been shown to be an important contributor to extreme sea levels, particularly along steep-shelved coastlines and narrow fringing reefs that characterize many small islands and atolls, e.g., [6-9]. In addition, whereas the cyclone-induced storm surge tends to be concentrated in the region of maximum onshore winds close to the cyclone center, wind-waves propagate with little loss of energy over the deep ocean, and so can increase the scale and duration over which damaging coastal impacts occur during a TC event [10].

McInnes et al. [11] applied a stochastic and numerical modelling approach to assess the frequency and magnitudes of storm tides around the Samoa archipelago under TC conditions. However, that study did not consider wind-wave processes and was regional in scale: extreme sea-level patterns below the scale of a kilometer were not resolved. An additional study, reported in Hoeke et al. [12], used a high-resolution storm surge model, coupled with a spectral (phase-averaged) wave model as well as a one-dimensional phase-resolved wave model, and inputs from McInnes et al. [11] to produce TC-induced inundation hazard information for the redevelopment of the Samoan parliament building, located in Apia (the capitol city of Samoa). In this study, we expand on these two earlier works by performing a series of additional simulations with the purpose of investigating the scale and spatial variation of the relative contribution of wind setup and wave setup to TC-induced extreme sea levels, as well as how these dynamics may change with SLR. This is accomplished by forcing the aforementioned high-resolution coupled wave and storm surge model of the Apia coastline with two sets (ensembles of 30 members each) of stochastically generated TCs. The two sets of cyclones produce storm tides near the statistical 50-year and 100-year recurrence interval (return period) levels at Apia, respectively, 
according to the findings of McInnes, et al. [11]. Three forcing "regimes" are considered: "wind", "wave", and "all". These forcing regimes allow the separate contribution of wind and wave setup, and how they interact, to be examined; the ability to separate forcing mechanisms is an advantage of numerical modelling exploited by past studies [13]. Two background sea-level scenarios are also considered: a "baseline" scenario which sets sea level to the mean local level between 1980 and 1999 relative to recently surveyed topography and bathymetry, and a "SLR" scenario where $1 \mathrm{~m}$ is added to the baseline scenario. The SLR scenario is not intended as a quantitative projection of local sea level rise, and no time horizon is assigned to it, although it is within the upper range of regional sea level projections for 2081-2100 [14]. The SLR scenario is instead a proxy to examine potential changes in wind and wave setup with an increased background sea level.

The coastal morphology of Apia (and of most of the Samoan Archipelago) is that of a basaltic island surrounded by fringing reefs, a common morphology of tropical and sub-tropical oceanic islands worldwide and may be considered representative of morphological analogues in the Indian and Atlantic, as well as the Pacific.

The remainder of the paper is organized as follows. The next section gives an overview of the study site, the methodological approach and the implementation of models that are used. Model results are presented in Section 3, followed by a discussion and conclusions in Sections 4 and 5, respectively.

\section{Experimental Section}

\subsection{Study Site and Context}

The larger islands of the Samoan Archipelago are volcanic high islands, surrounded by a relatively narrow littoral strip of complex fringing reefs, frequently incised with channels and embayments, often associated with terrestrial streams. Apia, on the northern shore of the island of Upolu (Figure 1), is the capitol city of the Independent State of Samoa and site of most of the nation's infrastructure.

Tides in Samoa are mixed semi-diurnal with mean daily range of $0.86 \mathrm{~m}$ and a typical spring tidal range of $1.3 \mathrm{~m}$. While the northern coast of Upolu is frequently impacted by both local trade wind seas and long-period swell associated with northern hemisphere mid-latitude storms, analysis of a 34-year wave hindcast [15] indicates that all historical wave events with greater than a five-year recurrence interval were associated with the passage of named tropical cyclones [12]. Thus, extreme wind-wave events impacting Apia are most likely generated by TCs.

Several tropical cyclones have affected Samoa in recent decades, including TC Ofa in 1990, TC Val in 1991, TC Tui in 1998, TC Heta in 2004 and most recently TC Evan in 2012. In February 1990, TC Ofa killed seven people and caused damages estimated at US\$130 million [16]. TC Val in December of the following year was more destructive due to the slow movement of the cyclone, causing 15 deaths, the loss of $95 \%$ of homes in Samoa and approximately US\$200 million damage $[17,18]$. The tide gauge at Apia was not installed until 1993 [19], so only anecdotal information is available on extreme water levels arising from the earlier cyclones. Much of the destruction during TC Ofa was caused by waves and high water levels [18]. Deep water wave heights during TC Val were reported to reach $7.5 \mathrm{~m}$ while maximum water levels were up to $1.6 \mathrm{~m}$ and resulted in the Apia Observatory (on the Mulinu'u Peninsula, Figure 1) being submerged to $0.5 \mathrm{~m}[20,21]$. These reports, though providing only qualitative 
comparisons to the modelling outputs for the most part, have been used to assess and validate the models and results presented in this study.

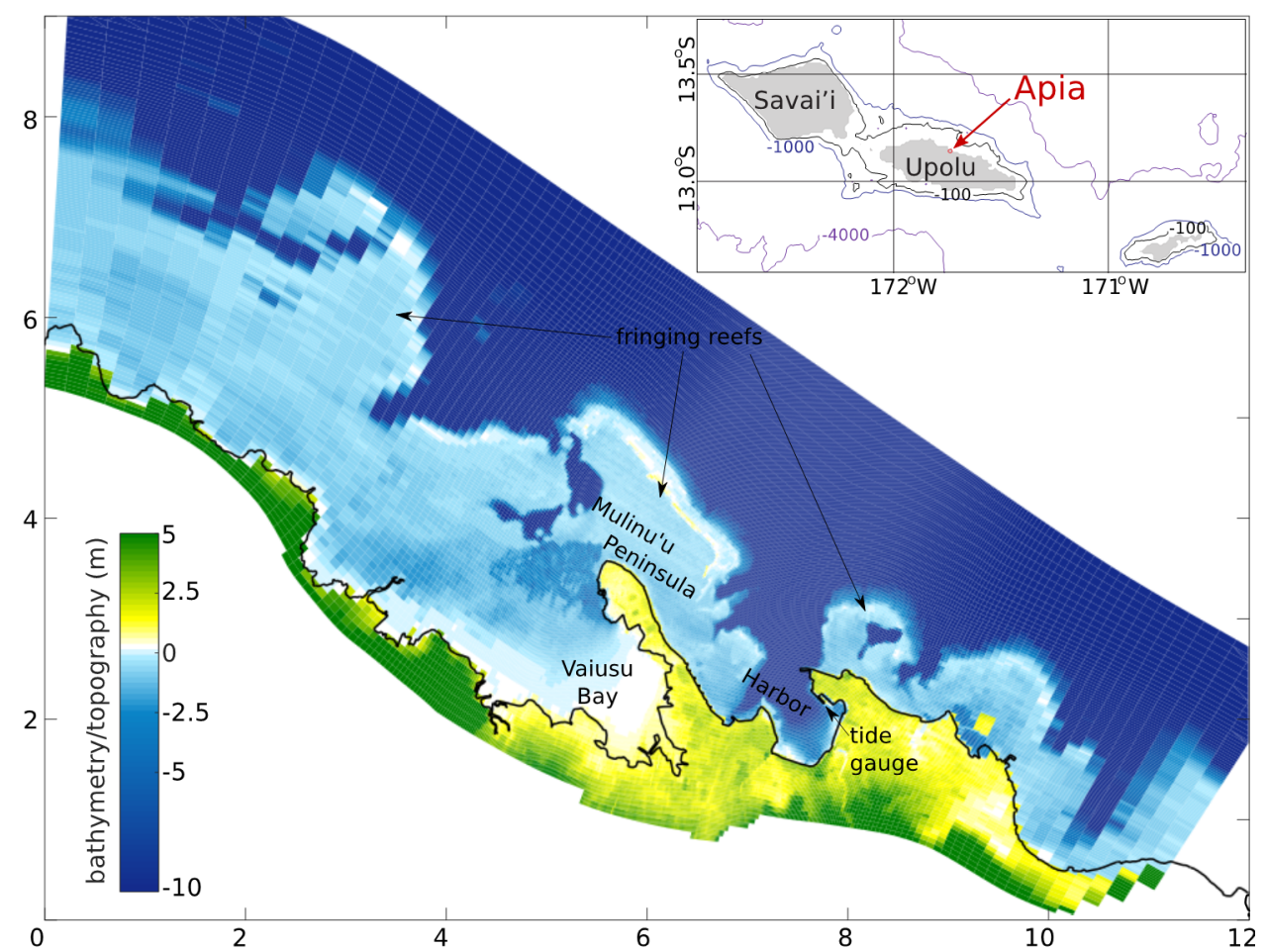

Figure 1. Location map of Apia in the Samoan Archipelago (inset, upper left) and the Apia Model's curvilinear grid and bathymetry/topography (main panel) with key geographical features labeled. Note grid resolution varies from approximately $100 \mathrm{~m}$ near the boundaries to approximately $10 \mathrm{~m}$ near the center. The numbers on the $\mathrm{x}$ - and $\mathrm{y}$-axes indicate distance in kilometers.

\subsection{Ensemble Methodology}

The TC ensembles used in this study are drawn from the stochastic cyclone and storm tide modelling study of McInnes et al. [11]. The methodology for generating and selecting cyclones, and subsequently forcing the (high-resolution) Apia model simulations, is illustrated schematically in Figure 2. Steps 1 to 3 summarise the approach used in McInnes et al. [11], in which synthetically- generated populations of tropical cyclone histories were developed for Samoa. Steps 1 to 3 of [11] are described briefly here for completeness. Step 1 involved analysing historical cyclones in an $8^{\circ}$ radius of Samoa; the large radius being to ensure sufficient events to develop empirical probability density functions (PDFs) for cyclone attributes of track, translation, speed, and intensity. The PDFs were then sampled to generate synthetic storm tracks. In step 2 these were used in conjunction with the analytical cyclone ("vortex") model described in Holland [22] to develop wind and pressure gridded fields for each synthetic storm track for a 1-km resolution archipelago-scale hydrodynamic model that simulated the water levels due to the resultant storm surge. An example of Holland vortex winds and the extent of the 1-km resolution archipelago-scale hydrodynamic model are given in Figure 3a. Astronomical tides were also included by randomly assigning to each storm track a commencement time during the cyclone season within a tidal epoch and simulating the associated tide variations to ensure random phasing of tides with each synthetic cyclone 
event. Analysis of the synthetically generated sea level maxima in step three involves assigning an average frequency of occurrence according to the observed cyclone frequency within the region covered by the model grid to develop storm tide recurrence intervals (RIs). The 50 and 100-year storm tide RIs were assessed to be $0.81 \mathrm{~m}$ and $0.92 \mathrm{~m}$ respectively [11].

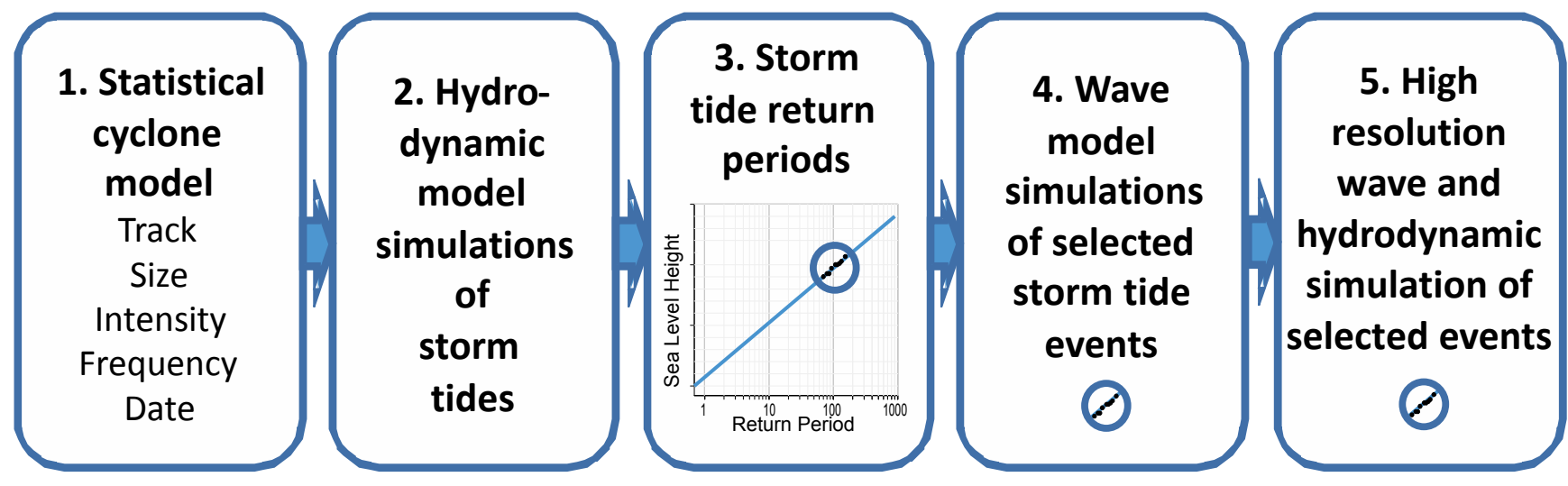

Figure 2. Schematic illustrates the method adopted in this study. Steps 1 to 3 relate to the study of McInnes et al. [11] in which synthetic cyclone and hydrodynamic models are used to estimate storm tide return periods. Steps four and five are undertaken in this study to evaluate the additional contribution from wave setup.

Given the large deep-water fetches and long period waves typical of Pacific TCs [2], the "wave" and "all" forcing regimes of the Apia model require wave modelling of a large ocean region to produce realistic wave boundary conditions (step four of Figure 2). The archipelago spectral wave model implemented to do this (see next section), as well as the wave module of the Apia model itself, are an order of magnitude more computationally expensive than the depth-integrated storm surge model at similar scales; therefore, simulating wave fields for the thousands of TC events generated in step one would be prohibitive for most practical applications. In this study the problem has been made tractable by selecting, from the total population described by McInnes [11], the 30 cyclones with maximum storm tide values nearest to the 50 and 100-year RI values at Apia, respectively. A total of 30 synthetic TC ensemble members was chosen because this (qualitatively) appeared to capture most probable cyclone approaches to the Apia shoreline for both the 50 and 100 year RI ensembles; these two RIs were selected because they are commonly of interest for engineering and planning applications. This "reduced ensemble" approach allows the range of variability in wave setup to be explored for a given storm tide level. As will be shown in the next section, not all parts of the coastline are exposed to wave setup; indeed the location of the tide gauge within the sheltered deep water harbour at Apia is one such location. Without a priori information on wave setup, this supports the two-step approach adopted in this study of first evaluating the storm tide RIs (i.e., no waves) from the full population of stochastic TCs, which are relevant for locations little affected by waves (e.g., the tide gauge), and second building in the additional contribution from the waves, through subsequent high-resolution modelling.

In summary, three different forcing regimes ("wind", "wave", and "all") are considered for each TC in the two 30-member ensembles, one corresponding to the 50-year RI storm tide levels and the other, to the 100-year RI levels, as defined by McInnes et al. [11]. Two different background sea levels are also considered: "baseline" and "SLR". This results in a total of 12 ensembles (each with 30 members), 
or 360 total simulations of the Apia model described in the next section (not including historical simulations used for model validation).
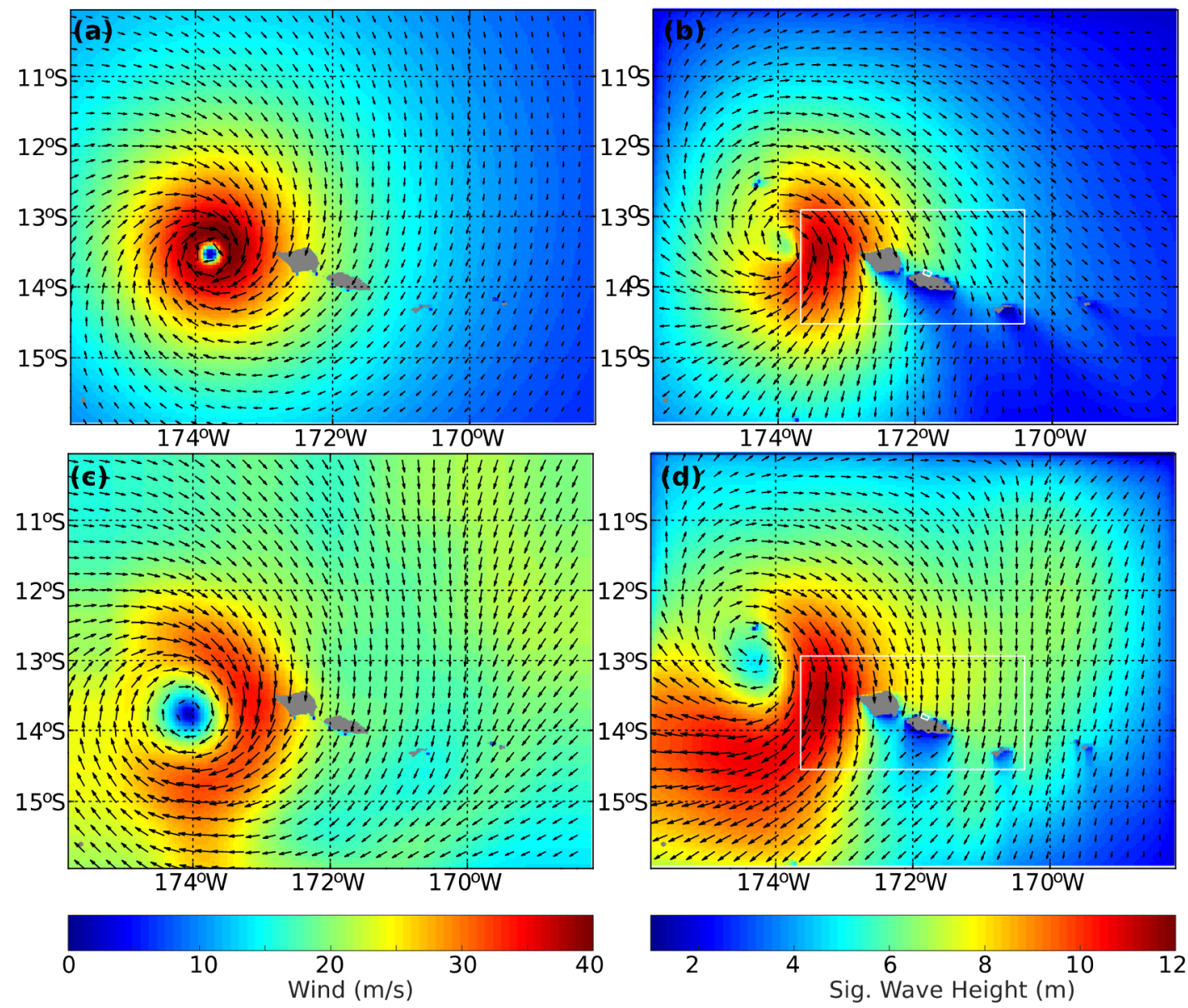

Figure 3. Example of Holland vortex winds (a) and simulated significant wave height and peak direction (b) during the historical TC Ofa (on 2 February 1990, 1200UTC). CFSR winds (c) and simulated waves (d) are included for comparison. The larger white box in (b) and (d) indicate the 1-km resolution nested SWAN model region, the smaller white box indicates the location and extents of the Apia Model.

\subsection{Model Implementation}

\subsubsection{Archipelago Wave Model}

In order to provide wave boundary conditions associated with the synthetic and the historic TCs, an archipelago-scale nested Simulating WAves Nearshore (SWAN) model (version 41.01) was implemented over the region. The SWAN model predicts the evolution in time and space of the wave action spectrum [23]; here, spectral resolution was as follows: 72 directional bins ( $5^{\circ}$ resolution) and 24 frequency bins, logarithmically spaced between 0.042 and $0.411 \mathrm{~Hz}$. A number of previous studies have shown SWAN's default bulk wind input formulation [24] may overestimate wind drag coefficients $\left(C_{d}\right)$ in tropical cyclone wind conditions [25,26]; $C_{d}$ is therefore capped at $2.5 \mathrm{~m}^{2} / \mathrm{s}$ and the wind source term implementation is set to that reported in Janssen [27]. 
The SWAN model's outer boundaries were the same as the modelling boundaries described in [11], i.e., that of the TC wind fields (Figure 3a). Spatial resolution was set to $5 \mathrm{~km}$, with a $1-\mathrm{km}$ resolution nest centred on the islands of Upolu and Savai'i (Figure 3b). This model was validated by comparing wave fields generated by the SWAN model using both synthetic (Holland vortex) and Climate Forecast System Reanalyses (CFSR, [28]) winds with a multi-year wave hindcast for the region [15] for a number of historical TCs (e.g., Figures 3 and 4). Comparisons between CFSR and Holland vortex wave fields are covered briefly in the results section of this paper; however the validation is presented at some length in [12].
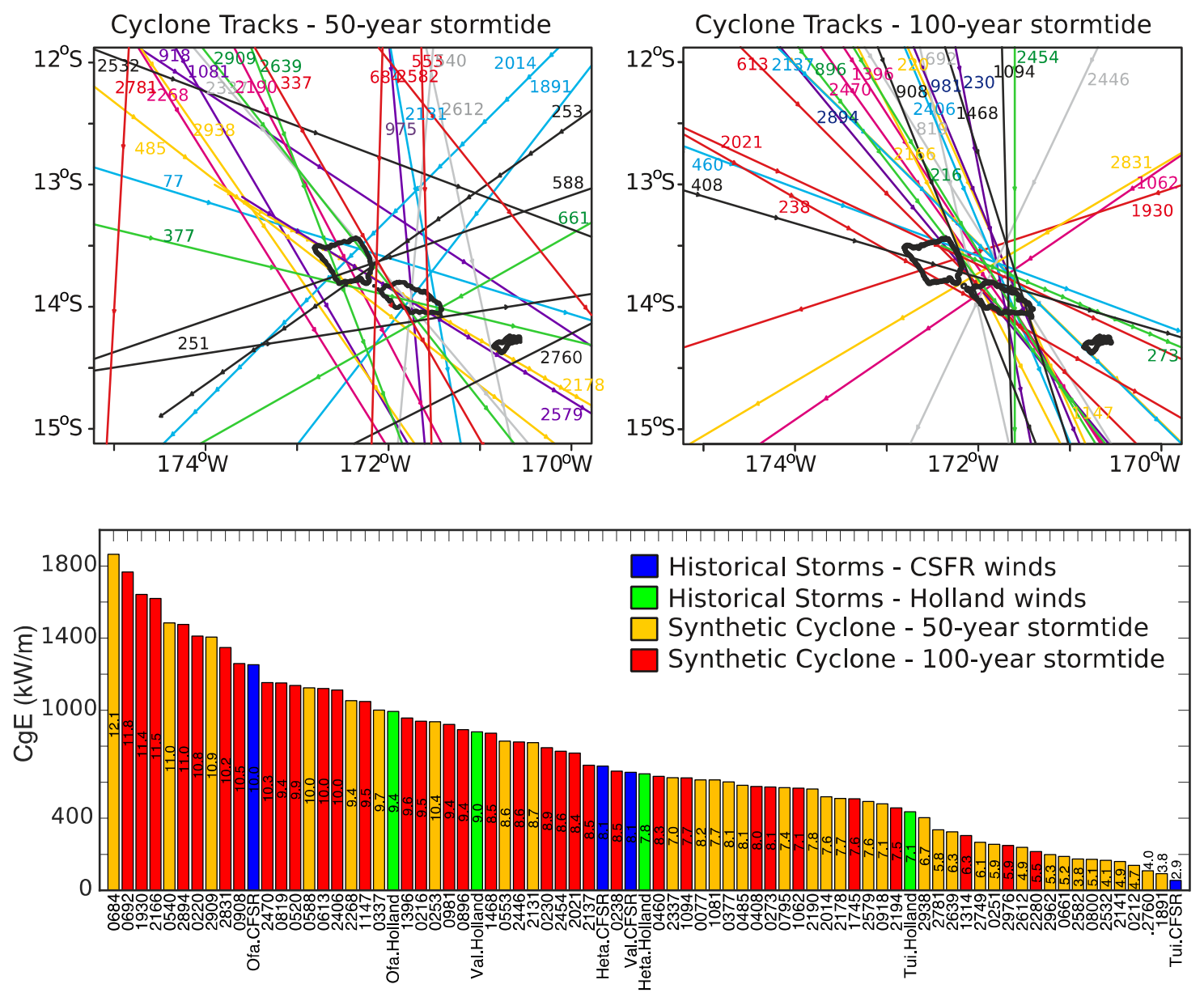

Figure 4. Upper panels: synthetic cyclone tracks used in this study: selected 50-year RI ensemble (left) and 100-year ensemble tracks (right). Lower panel: maximum wave energy flux $\left(C_{g} E\right)$ calculated by the SWAN model at a point just north of the Apia Model's boundary for each of the events modeled in this study; labels on the bars indicate corresponding significant wave height.

\subsubsection{Apia Model}

For the simulation of extreme sea levels around Apia during TC conditions, the open-source version (6.01.15.5013) of the Delft3D flow-wave-coupled hydrodynamic modelling system [29] was used. This system has been widely applied to combined storm surge/wave setup processes, e.g., [30,31] and has been successfully applied to tropical fringing-reefs such as those found in Samoa, e.g., [32,33]. 
This system's "flow" module consists of a finite-difference solution to the Navier-Stokes equations for unsteady flow; the "wave" module is the previously described SWAN model. The two modules are iteratively coupled so total dissipation forces (radiation stresses) from the wave module are passed to the flow module to compute wave-induced residual flow and Stokes drift; the subsequent water levels and currents in the circulation module are passed back to the wave module to calculate an updated wave field. The Delft 3D model implementation used here (called the Apia model henceforth) was set up on a 2D curvilinear grid, which varied in spatial resolution from approximately $200 \mathrm{~m}$ near the northwest and southeast (lateral) boundaries, to approximately $10 \mathrm{~m}$ near the Mulinu'u Peninsula and Apia Harbor (Figure 1). This spatial resolution was made feasible by a high-resolution light detection and ranging (LiDAR) topography and bathymetry survey of the Apia area.

Astronomical tidal predictions, based on the Apia tide gauge, and background sea level were combined with linearly interpolated inverse barometer effect (IBE) from the archipelago storm tide model to produce a spatially- and temporally-varying offshore water level boundary; shore-normal lateral boundaries were calculated as water level gradients, i.e., Neumann boundary conditions [34]. Wave boundary conditions were supplied as directional spectra from the Archipelago wave model at points along the Apia model boundary; spectral resolution and source terms were set the same as for the archipelago SWAN model. Wave dissipation and subsequent wave setup over reefs have been shown to be sensitive to hydraulic bed roughness $\left(f_{w}\right)$ and the wave breaking index $\left(\lambda_{b}\right)$, defining the water depth at which waves will break, i.e., $H_{b}=h \lambda_{b}$ where $H_{b}$ is breaking wave height, $h$ is water depth. In keeping with a number of studies $[8,33,35,36]$, we implement a spatially-varying $f_{w}$ grid with roughness lengths equivalent to $0.15 \mathrm{~m}$ in reef areas and 0.02 in sandy areas [37], and set the $\lambda_{b}$ to 1.0 based on mean fore-reef bottom slope according to the empirical relationship of Raubennheimer [38].

Water level observations at the Apia tide gauge and a nearby 11-month long mooring deployment were well correlated $(R \geq 0.98)$ with those predicted by the Apia model; root mean square errors (RMSE) were less than $0.10 \mathrm{~m}$ at those locations. Reports of inundation extent and damage correspond well with that modelled, particularly for Cyclone Ofa, which experienced the worst coastal inundation of recent decades. Further details on validation, model parameters and other information are detailed in Hoeke et al. [12].

The three different forcing regimes were all simulated using the "flow" module with the same water level boundary forcing: in the "wind" regime the flow module was not coupled to the "wave" module. Thus, no wave radiation stresses were present; in the "wave" regime, the flow module was coupled to the wave module but assumed a surface wind stress of zero; in the "all" regime both wind and wave forcing was present.

\section{Results}

\subsection{Archipelago Wave Model Results}

Wave model simulations were performed for each member of the two synthetic TC ensembles (corresponding to 50 and 100-year storm tide RIs). The tracks of the cyclones (Figure 4: upper panels), indicated that those contributing to the 50-year storm tide levels were spread across the archipelago, while those producing the 100-year storm tide levels crossed in much closer proximity to Apia, 
consistent with these events leading to higher coastal sea levels through inverse barometer effect and wind setup (storm surge). An example of the modelled wind and wave field during a synthetic event is shown in Figure 3a,b. Maximum deepwater wave energy flux $\left(C_{g} E\right.$, i.e., the power available to drive wave setup and other shallow water wave processes) for all ensemble members for a point on the Apia model's offshore boundary is shown in Figure 4 (lower panel). These maxima exhibit a broad range of values across the two ensemble members (60 storms in total); corresponding significant wave heights $\left(H_{s}\right)$ range from 3.8 to $12.1 \mathrm{~m}$. This upper range is within the empirical values found by Stephens and Ramsay [2] for Samoa: 10.6-13.7 m and 11.6-15.3 m for 50 and 100-year RI, respectively. The smaller values of many of the ensemble members found in this study is most likely due to the fact that Stephens and Ramsay [2] did not include any wave sheltering effects (e.g., of islands), whereas here they are dynamically included. The ranking of ensemble members by $C_{g} E$ in Figure 4 (lower panel) indicates that while maximum wave heights generated by the 100-year ensemble are generally higher than those within the 50-year ensemble, this is not necessarily always the case; the maximum overall $C_{g} E$ value in Figure 4 is actually from a member of the 50-year ensemble. This is a reflection of the fact that factors besides TC proximity to Apia and wind strength are important in determining maximum $C_{g} E$ impingent on Apia, i.e., TC track orientation, radius of maximum winds and propagation speed. Furthermore this indicates that local maximum $C g E$ is not necessarily well correlated with the local storm surge level (i.e., IBE and wind setup) simulated by McInnes et al. [11].

Historical TC waves simulated with either CFSR winds [28] or Holland vortex wind fields are also included in Figure 4 (lower panel) for comparison; these are scattered amongst the ensemble maxima, indicating no consistent bias in maximum $C_{g} E$ between the synthetic TC approach and various historical approaches. In particular, the position of the TC Ofa's maximum $C_{g} E$ in the middle (synthetic forcing) to upper end (CFSR forcing) of the rankings is broadly consistent with the TC's characteristics (radius of maximum winds and central pressure) relative to other historical TC information used to construct the synthetic cyclone population. However, as noted by a number of studies (e.g., [39,40]), CFSR wind fields may not be of sufficient spatial resolution to accurately simulate extreme waves near the TC eye wall; conversely synthetic vortex wind fields may underestimate far-field winds (and thus wave generation) by neglecting to take into account larger-scale meteorological conditions. An example of this is evident in Figure 3: an area of stronger winds and elevated $H_{s}$ to the east of TC Ofa is evident in the CFSR simulation (Figure 3c,d) relative to the Holland vortex simulation (Figure 3a,b). Several researchers have attempted to ameliorate such issues by blending reanalysis winds with vortex winds (e.g., [40]); this is of course not an option for the synthetic cyclones used in this study, however. Sensitivity of extreme wave prediction to different input wind fields remains poorly understood; something exacerbated by an almost total lack of in situ TC wind and wave records in the insular Pacific [2,7]. Further consideration of this is beyond the scope of this study, however, and remains an area of future research. Comparisons of historic and synthetic cyclones and differences in wind forcing are discussed at greater length in $[3,11,12,39]$.

\subsection{Apia Model Results}

Apia model simulations were typified by rapid wave dissipation through wave breaking and bed friction across the fore-reef slope of the fringing reefs, resulting in wave setup over the shoreward reef 
flats and rapid offshore return flow (on the order of $1 \mathrm{~m} / \mathrm{s}$ ) in reef passes and channels (Figure 5). This pattern is consistent with a number of studies at other locations, e.g., [13,33,41,42]. Figure 5 also shows the position of four locations ("offshore", "reef", "bay", and "tide") that are discussed in more detail below. It is noteworthy that the location of the tide gauge ("tide") in the harbour is relatively sheltered from both high waves and wave setup.
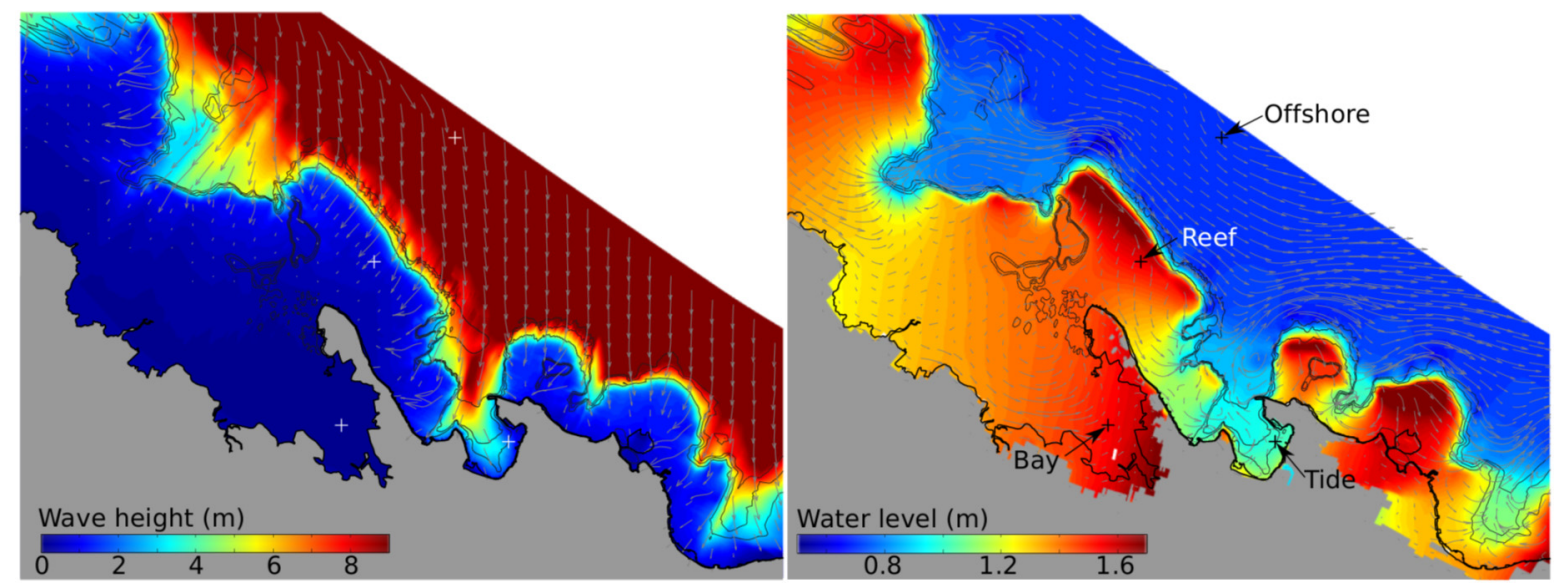

Figure 5. Apia Model output near the peak of local water levels during Cyclone Ofa. (Left panel): significant wave height and peak wave direction (grey arrows). (Right panel): water levels and depth-averaged current vectors (grey arrows). Locations discussed in the text are labelled in the right panel.

The spatial water level maxima of the Apia model simulations in the two TC ensembles exhibit a high degree of alongshore and cross-shore variability across all three forcing regimes ("wind", "wave", and "all"). These spatial patterns can be summarized by the median and 95th percentile values of the individual ensemble maxima; these are plotted in Figures 6 and 7 for the 100-year RI baseline and 100-year RI SLR scenario, respectively. (The spatial patterns of 50-year RI ensembles were similar albeit lower in height so are not plotted for brevity). Median values of the 100-year RI baseline ensemble range spatially between 0.9 and $1.9 \mathrm{~m}$, except for wind forcing, which has a lower upper range, around $1.4 \mathrm{~m}$ (Figure $6 \mathrm{a}-\mathrm{c}$ ). The low end of the median values occur near the "tide" location in the harbour and are consistent with the 100-year RI for storm tide values $(0.92 \mathrm{~m})$ determined by McInnes et al. [11]. These results therefore support the idea that storm tide-only values (such as presented in McInnes et al. [11]) are relevant for the "tide" location, which is largely sheltered from wave-induced sea level contributions and local wind setup, but that large local excursions from this "reference" value occur due to bathymetric and shoreline variations in the local vicinity, which are accounted for in the high-resolution modelling. 95th percentile values from the baseline, 100-year RI ensemble vary between roughly 1.1 and $2.5 \mathrm{~m}$ for all three forcings (Figure 6d-f). While the ranges of ensemble values across the three forcings are similar, their spatial patterns are not. The wind forcing regime in particular shows that locally elevated water levels relative to the rest of the model grid are largely restricted to Vaiusu Bay (Figure 6a,d). Wave setup is significant throughout much of the grid in the wave forcing case, especially near the reef crest offshore from the Mulinu'u Peninsula, where values are $1 \mathrm{~m}$ or higher than elsewhere in the grid (Figure 6b,e); while lower than at the reef crest, wave forcing exhibits elevated water levels (relative to wind forcing) over much of the reef flat areas and Vaiusu Bay. Simulated "all" forcing regime water levels are 
qualitatively a combination the wind and wave forcing regime water levels (Figure $6 \mathrm{c}, \mathrm{f}$ ), though not a linear superposition of the two, particularly in areas close to shore. Reasons for this are discussed in the following sections. Patterns of the median and 95th percentile maximum water level values for the 100-year RI ensemble that includes the SLR scenario (Figure 7) are quite similar to the baseline, 100-year RI scenario, except being approximately $1 \mathrm{~m}$ higher. Here too, however, there are some significant departures from a simple linear increase between the two, particularly over the reef flat and Vaiusu Bay areas. This is also discussed in more detail in the following sections.

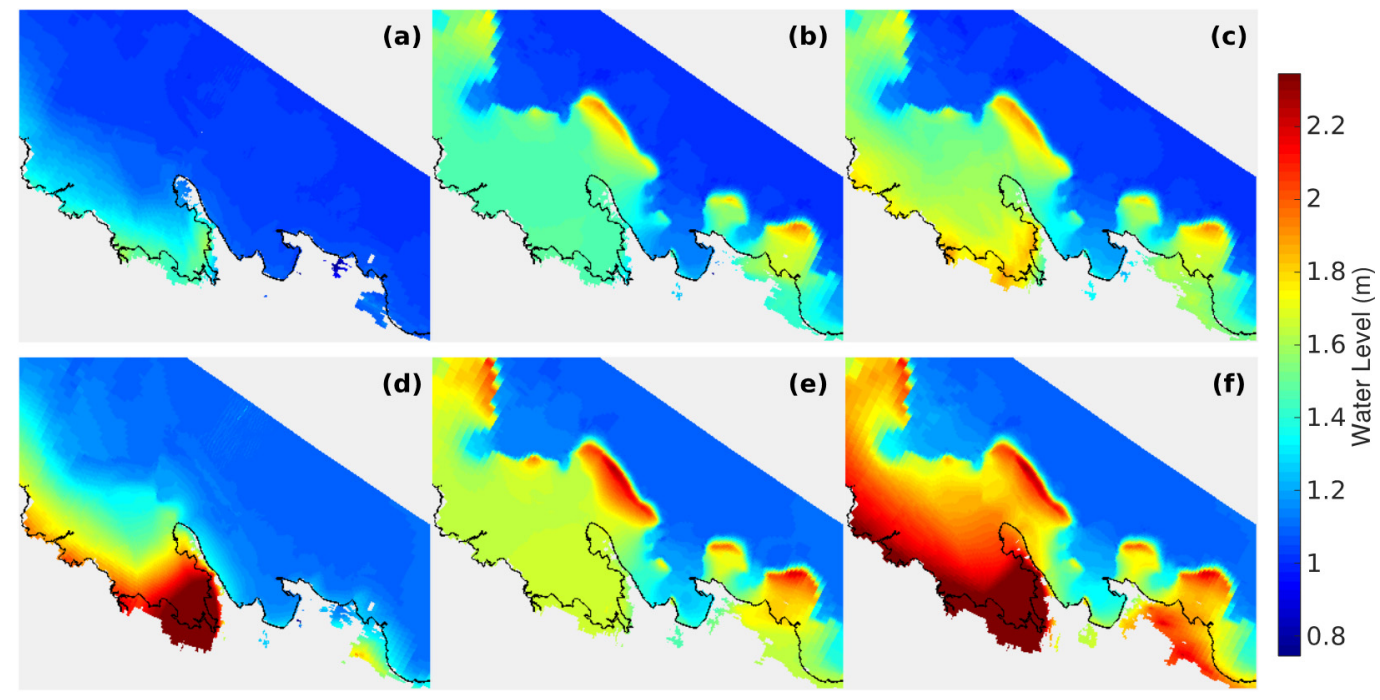

Figure 6. Water level maxima for the 100-year RI ensemble, "baseline" scenario. Median ensemble values for "wind" forcing (a); "wave" forcing (b); and "all forcing" (c). Subplots $(\mathbf{d}-\mathbf{f})$ are the same as $(\mathbf{a}-\mathbf{c})$ ensemble except are 95 th percentile ensemble values.

It is apparent there is a large departure between the median and 95th percentile values within Vaiusu Bay for wind forcing (Figures 6a,d and 7a,d) relative to other areas/other forcing. This is due in part to the high sensitivity of water levels within the bay to TC track: slight changes in maximum wind direction lead to large changes in local wind setup due to the bay's morphology. In fact, different areas within the grid show highly varying sensitivity to the different forcing regimes. This is summarized in Figure 8, which plots the range of water level maxima amongst ensemble members for each of all ensembles at the four locations ("offshore", "reef", "bay", and "tide") defined in Figure 5. The total range (variance) of maximum water levels among the ensemble members is relatively low, with only small differences between the forcing regimes not only at the offshore location (which would be expected), but also at the tide (gauge) location. At the reef location however, it is low only for the wind forcing regime; the wave and all forcing regimes exhibit more than three times the variance of the wind forcing, indicating the dominance of wave setup on extreme sea level processes at this location. At the bay location (within Vaiusu Bay), however, wind forcing ensembles exhibit a larger range of maximum water levels than the wave forcing ensembles at the "reef" location; closer inspection of these "wind" values, relative to the surrounding coast, reveals a strong relationship to the track of the TCs. Simulations that produced local maximum sea levels in the southern part of Vaiusu Bay tend to be those associated with north-to-south TC tracks and crossing in the immediate vicinity or to the west of Apia, maximising the northerly winds on the eastern flank of the cyclone (e.g., see 50-year RI tracks 684, 975, 2909, and 2190 and 100-year 
RI tracks 613, 692, 896, 908, 981, 1396 in Figure 4). In the simulations in which maximum setup occurred on the western side of Mulinu'u peninsula, the cyclones typically tracked from north to south on the eastern side of Apia (e.g., 50-year RI tracks 553 and 2131 and 100-year RI tracks 819, 981, and 2454) and produced strong westerly winds after the cyclone centre crossed Samoa. Water levels in Vaiusu Bay tended to be lower than surrounding coastal region (e.g., wind setdown occurred) for fast moving cyclones that followed a more east-west trajectory to the north of Samoa (e.g., 100-year RI tracks 460 and 1147) while cyclones whose track was at a greater distance from Samoa (e.g., 50-year RI track 1081) did not produce significant wind setup in Vaiusu Bay. Conversely, wave setup values across the Apia model domain did not exhibit nearly such local sensitivity to details of cyclone track; these values were more dependent on TC radius of maximum winds and central pressure.

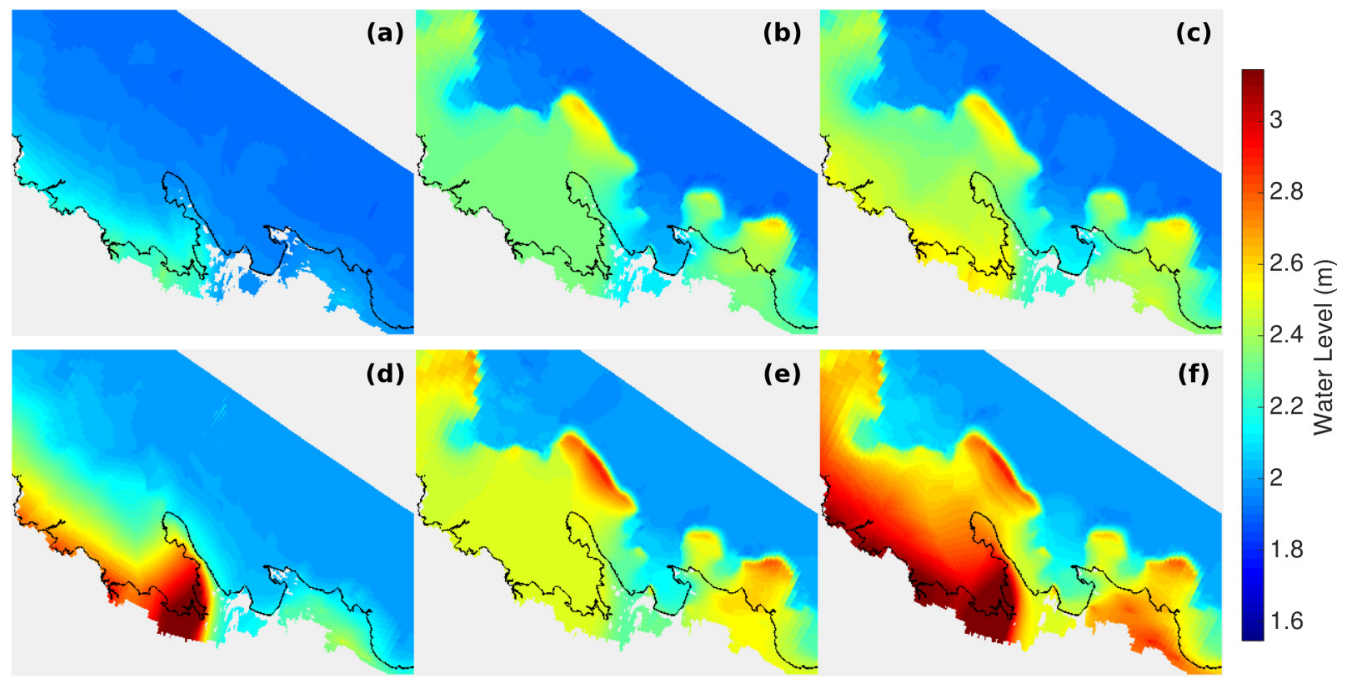

Figure 7. Water level maxima for the 100-year RI ensemble, "SLR" scenario. Median ensemble values for "wind" forcing (a); "wave" forcing (b); and "all forcing" (c); Subplots $(\mathbf{d}-\mathbf{f})$ are the same as $(\mathbf{a}-\mathbf{c})$ ensemble except are 95 th percentile ensemble values.

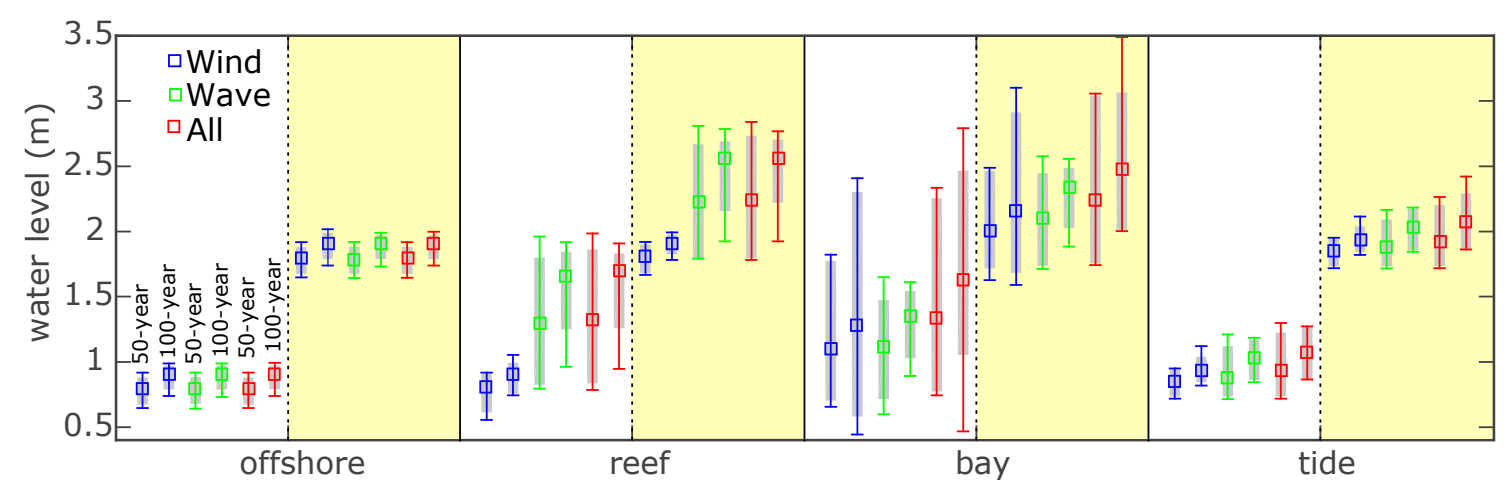

Figure 8. Distribution of maximum water levels amongst 50-year and 100-year RI ensemble members at "offshore", "reef", "bay", and "tide" locations (see Figure 5). Blue, green, and red indicate wind, wave, and all forcing, respectively (as indicated at upper left); median ensemble values are indicated with a square; 5 th and 95 th percentile values are indicated with grey bars, respectively, and ensemble minima and maxima by colored error bars. At each location indicated, the "baseline" scenario is on the left (white background) and the "SLR" scenario is on the right (yellow background). 


\section{Discussion}

The results indicate that the amount of wind setup and wave setup varies locally by up to approximately a meter for both 50 and 100-year RI cyclones. Wave setup tends to be the dominant process, particularly at coastal locations adjacent to shore-parallel reef crests such as the Mulinu'u Peninsula. At such locations, the results presented here closely approximate a number of analytic/empirical solutions of wave setup which assume straight and parallel alongshore topography and wave conditions, e.g., $[8,43,44]$. In particular, water levels within several hundred meters shoreward of the reef crest fronting the Mulinu'u Peninsula (e.g., the "reef" location) simulated by the wave forcing ensemble are within approximately 10 centimetres of the solution described by Becker et al. [44], which is plotted for comparison with model output in Figure 9. Closer to shore, or at other locations without a neighbouring shore parallel reef crest of some length, these analytic methods greatly overestimate wave setup. This would be expected, since these solutions generally assume a complete momentum balance between wave radiation stress and pressure (water level) gradient, whereas the 2-dimensional numerical solution presented here allows for return flow dependent on morphological details and bed roughness, as would realistically be the case, particularly in the complex reef morphology typical of many tropical high islands.

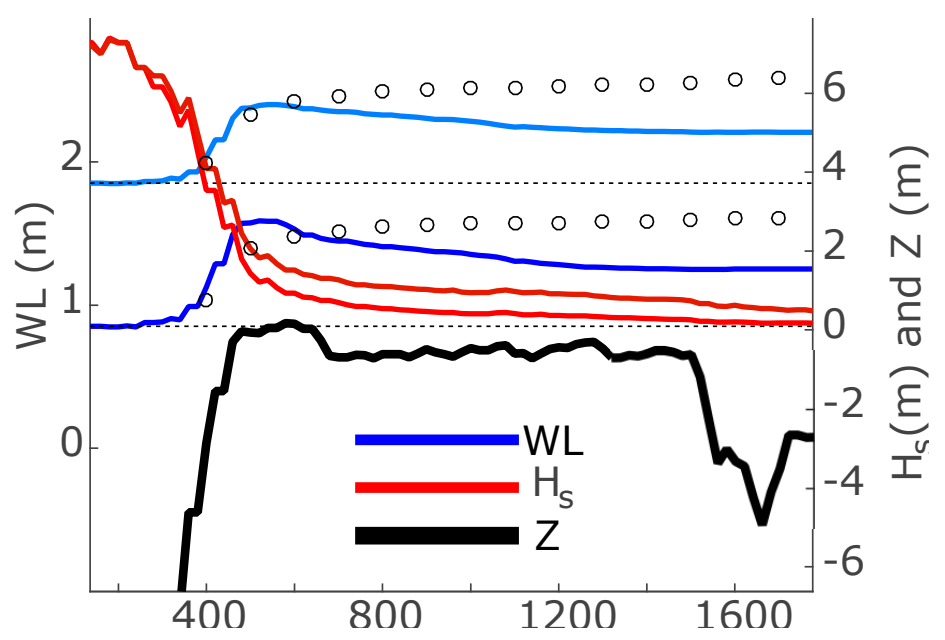

Figure 9. Ensemble (wave forcing, baseline, and SLR scenarios) significant wave heights $\left(H_{s}\right.$, red) and water levels (WL, blue), along a transect through the "reef" location (shown in Figure 5); offshore is on the left side, landward direction towards the right; topography $(Z)$ is indicated with a black line. The analytic estimate of wave setup described by Vetter et al. [8] and Becker et al. [44], based on $H_{s}$ from the Apia model, is plotted with black circles, with the caveat that breaking parameter $\lambda_{b}$ is set to 1.0 (consistent with the Apia model), rather than varying with water level.

While wave setup is the largest contributor to extreme water levels at most locations, that is not the case everywhere. This can be visualized by subtracting the wind forcing simulations from the wave forcing. Wave forcing is clearly dominant on the reef flats adjacent to exposed reef crests (negative values in Figure 10a), however, the high positive values in Figure 10a indicate the dominance of wind setup in Vaiusu Bay. Assuming that these processes are sufficiently locally independent so that separate (uncoupled) models for wind setup and wave setup can be combined (as may be desired) requires caution 
however. Adding the wind and wave forcing results leads to an overestimate of water levels relative to the "all" forcing in some areas, particularly near the shoreline (Figure 10b). This is primarily due to the increasing overall water levels (and thus depths) associated with the "all" forcing, which effectively decreases depth-integrated hydraulic roughness, allowing for greater volume transport of wind and wave setup offshore, resulting in decreased water levels relative to an independent combination of the two. This is also a reason that local increases in extreme sea levels are approximately $10 \%-30 \%$ less relative to background increases in sea level in the SLR versus the baseline scenario (Figure 11a), although decreased wave breaking (radiation stress) at the reef crest may also contribute. While this is potentially good news for coastal communities such as Apia, as it ameliorates the effect of extreme sea levels under conditions of SLR, it should be noted that this is accompanied by a relatively large increase in wave energy reaching the inner reef areas and the coastline as shown in Figure 11b; this is also visible in the higher SLR wave heights (compared to the baseline scenario) across the reef flat plotted in Figure 9.
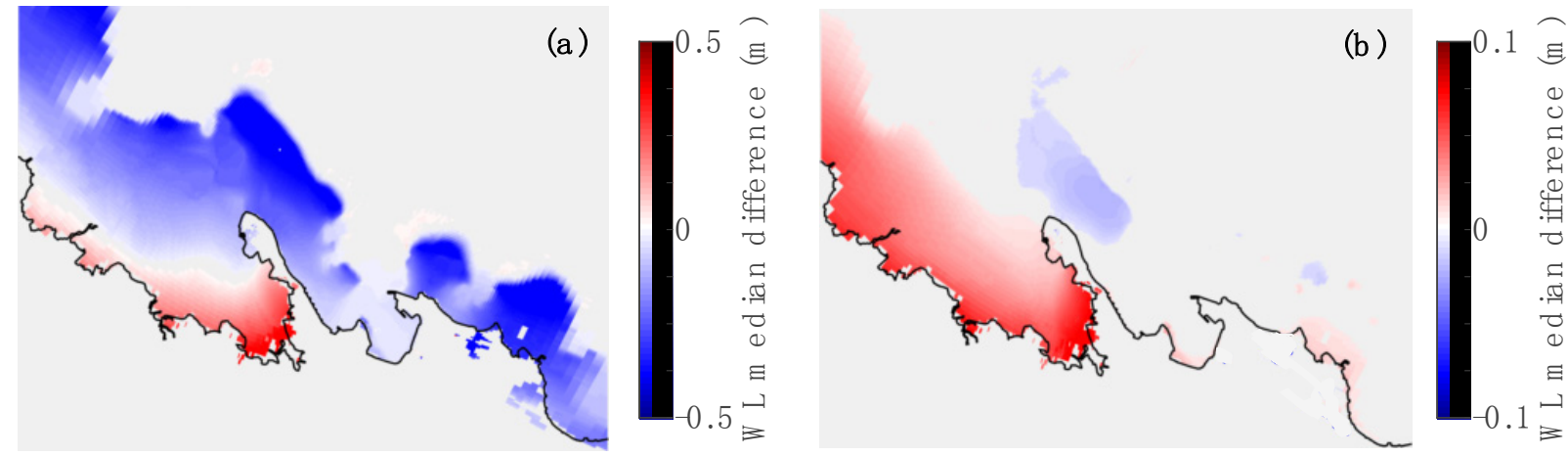

Figure 10. Differences in maximum water levels between "wind", "wave" and "all" forcing. (a): median difference between wind and wave maximas (wind minus wave); (b): median differences between the sum of wind and wave maximas (with a correction to prevent double counting tides and inverse barometer) and "all" forcing maximas.
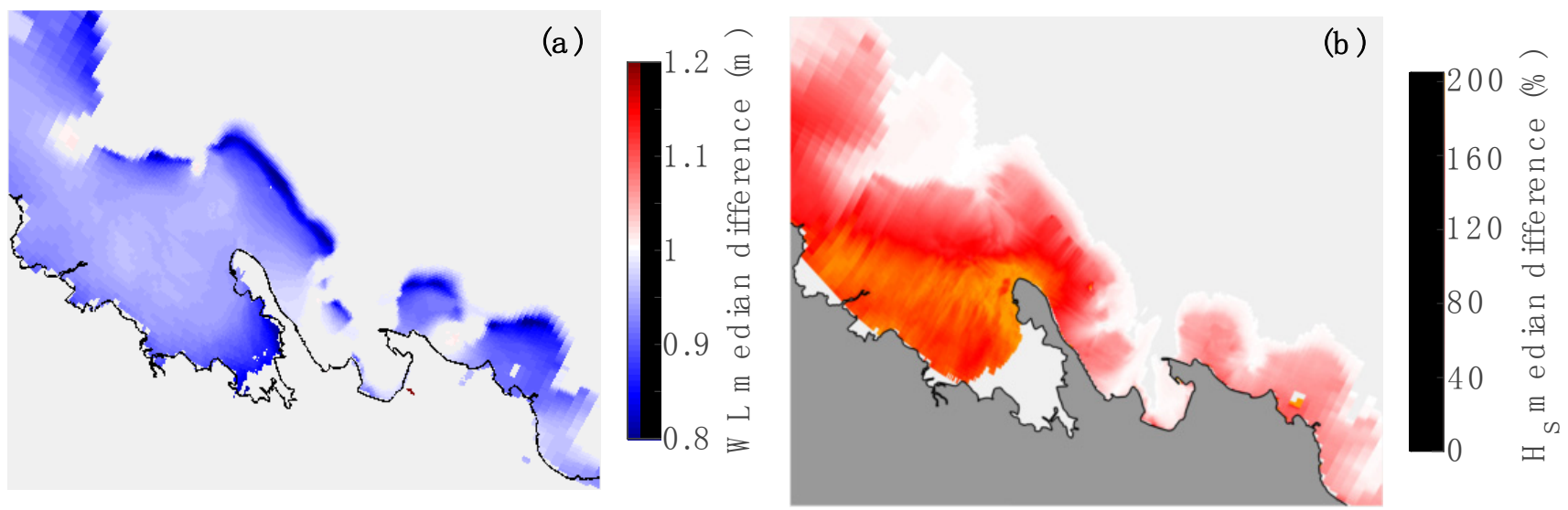

Figure 11. (a) Median differences between maximum water levels (WL) of the "SLR" and "baseline" ensemble members ("all" forcing); (b) median percent difference between maximum significant wave height $\left(H_{s}\right)$ of the "SLR" and "baseline" ensemble members ("all" forcing). In (b) areas with median $H_{s}$ less than $0.05 \mathrm{~m}$ or absolute differences of less than $5 \%$ are not plotted. 


\section{Conclusions}

In this study, high-resolution numerical simulations of coupled storm surge, tides, and waves, forced by tropical cyclone (TC) ensembles drawn from a much larger stochastically generated population [11], were used to examine the overall local variance of extreme sea levels and the relative contribution of wind and wave setup. Local water level excursions were found to be very sensitive to local morphology and storm track, with median extreme sea levels (and total ranges) for each ensemble differing by a factor of approximately two or more within less than a kilometre. Wave setup dominates extreme sea level processes in many areas, meaning analyses that do not include wave processes may significantly underestimate extreme sea level likelihoods in such settings. Crucially, both wave and wind setup effects are minimal at some locations, such as near the Apia tide gauge. This is an illustration of how poorly the likelihood of coastal inundation predicated on tide gauge data may represent the coastal areas in the immediate vicinity, particularly in areas that lack continental shelves (e.g., oceanic islands such as those of Samoa), something noted by a number of previous studies $[6,7,9]$.

To maintain tractable computational costs, a reduced member ensemble strategy was used in this study. Ensemble members were a priori based on cyclones that approximated the 50 and 100-year recurrence intervals (RI) storm tides at the Apia tide gauge, calculated from the archipelago-scale study of McInnes et al. [11], which did not consider the effects of wind-waves. While this approach is supported by the finding that both studies produced similar storm tide values near the tide gauge site (and other sites little effected by wave setup), the much larger overall spread of water level maxima among ensemble members at neighbouring reefs (such as fronting the Mulinu'u Peninsula, Figure 1, Figure 8) found in this study indicates that local incident $C_{g} E$ (and, thus, wave setup) is not necessarily well correlated with the local storm surge level (i.e., IBE + wind setup). This suggests that the same ensemble of TCs may not approximate the same local RI water levels at neighbouring locations. Therefore, future studies which utilize a pragmatic hybrid-downscaling approach similar to that used here would benefit from further investigation of the relationship between wind- and wave-induced sea level extremes, particularly when extrapolating to probabilistic statistics.

Comparisons of the high-resolution simulations show that $1 \mathrm{~m}$ of SLR relative to constant topography reduces the relative amount of combined wind and wave setup (on the order of $10 \%-20 \%$ ) but leads to a large increase in nearshore wave energy (up to approximately 200\%) in many areas. This is primarily due to decreased wave dissipation on outer reefs and is consistent with a number of other studies $[32,44]$. Seasonal and interannual sea-level variability, which is on the order of $30 \mathrm{~cm}$ at many Pacific islands, most likely similarly affects momentum balances in many locations. These local changes in momentum balances will result in changed patterns of inundation and sediment transport, particularly under progressively changing sea level. A problem with the assumption of static (constant) topography used in this and past studies is that it is probably unrealistic; however assuming alternate trajectories is far from straightforward as future changes in reef calcification rates remain unclear $[44,45]$ and synergistic effects between water quality and sea level are likely, e.g., [46]. Changes in these processes may lead to large changes in reef morphology, complexity, and roughness, which would then also lead to large local changes in momentum balances. This is an area of future research important to understanding the future effectiveness of reefs as natural coastal defences in island nations. 
Another important caveat is that the phase-averaged wave modelling in this and similar studies does not resolve individual waves or wave groups. As such it cannot resolve processes such as wave run-up, overtopping and infragravity frequency motions [47], which may be on the same order of magnitude as (sea-swell frequency) wind-waves near the shoreline in fringing reef systems $[9,40,48,49]$. Thus, transient extreme sea levels (e.g., time scales less than $30 \mathrm{~min}$ ) and the extents of coastal inundation are likely to be significantly underestimated in some areas. Phase-resolved numerical simulation of these motions is yet again an order of magnitude more computationally expensive than the phase-averaged models used here. This highlights the importance of good techniques to minimize (reduce) the number of ensemble members for computationally efficient, yet statistically representative, dynamic downscaling simulations. Improving these statistical techniques, as well as increasing computational capacity and development of more efficient numerical approaches at scales relevant to coastal impacts, is essential to facilitate greater usage of stochastic prediction methods, such as those presented here.

\section{Acknowledgments}

Much of the work reported here was supported by CSIRO and the Pacific-Australia Climate Change Science Adaptation Planning program (PACCSAP, www.pacificclimatechangescience.org/), in particular the Samoa Parliament Complex Redevelopment Project. The authors would also like to thank Felix Lipkin and Frank Colberg who contributed to various aspects of this study and CSIRO's Advanced Scientific Computing group for their excellent support. This manuscript was improved by the comments of three anonymous reviewers.

\section{Conflicts of Interest}

The authors declare no conflict of interest.

\section{References}

1. Diamond, H.J.; Lorrey, A.M.; Knapp, K.R.; Levinson, D.H. Development of an enhanced tropical cyclone tracks database for the southwest Pacific from 1840 to 2010. Int. J. Climatol. 2011, 32, 2240-2250.

2. Stephens, S.A.; Ramsay, D.L. Extreme cyclone wave climate in the Southwest Pacific Ocean: Influence of the El Niño Southern Oscillation and projected climate change. Glob. Planet Chang. 2014, 123, 13-26.

3. McInnes, K.L.; Walsh, K.J. E.; Hoeke, R.K.; O’Grady, J.G.; Colberg, F.; Hubbert, G.D. Quantifying storm tide risk in Fiji due to climate variability and change. Glob. Planet Chang. 2014, 116, 115-129.

4. Pugh, D.T. Changing Sea Levels: Effects of Tides, Weather and Climate; Cambridge University Press: Cambridge, UK, 2004.

5. Hubbert, G.D.; Mclnnes, K.L. A Storm Surge Inundation Model for Coastal Planning and Impact Studies. J. Coast. Res. 1999, 15, 168-185. 
6. Kennedy, A.B.; Westerink, J.J.; Smith, J.M.; Hope, M.E.; Hartman, M.; Taflanidis, A.A.; Tanaka, S.; Westerink, H.; Fai, K.; Smith, T.; et al. Tropical cyclone inundation potential on the Hawaiian Islands of Oahu and Kauai. Ocean Model. 2012, 52-53, 54-68.

7. Hoeke, R.K.; Mcinnes, K.L.; Kruger, J.C.; Mcnaught, R.J.; Hunter, J.R.; Smithers, S.G. Widespread inundation of Pacific islands triggered by distant-source wind-waves. Glob. Planet Chang. 2013, $108,128-138$.

8. Vetter, O.; Becker, J.; Merrifield, M.; Pequignet, A.; Aucan, J.; Boc, S.; Pollock, C. Wave setup over a Pacific Island fringing reef. J. Geophys. Res. 2010, 115, 1-13.

9. Merrifield, M.A.; Becker, J.M.; Ford, M.; Yao, Y. Observations and estimates of wave-driven water level extremes at the Marshall Islands. Geophys. Res. Lett. 2014, 41, 7245-7253.

10. Walsh, K.J.E.; McInnes, K.L.; McBride, J.L. Climate change impacts on tropical cyclones and extreme sea levels in the South Pacific-A regional assessment. Glob. Planet Chang. 2012, 80-81, 149-164.

11. McInnes, K.L.; Hoeke, R.K.; Walsh, K.J.E.; O’Grady, J.G.; Hubbert, G.D. Application of a Synthetic Cyclone Method for Assessment of Tropical Cyclone Storm Tides in Samoa. Nat. Hazards 2015, 79, doi:10.1007/s11069-015-1975-4.

12. Hoeke, R.; McInnes, K.; O’Grady, J.; Lipkin, F.; Colberg, F. High Resolution Met-Ocean Modelling for Storm Surge Risk Analysis in Apia, Samoa; CAWCR: Melbourne, Australia, 2014; Volume 71, pp. 1-80.

13. Mulligan, R.P.; Hay, A.E.; Bowen, A.J. Wave-driven circulation in a coastal bay during the landfall of a hurricane. J. Geophys. Res. 2008, 113, doi:10.1029/2007JC004500.

14. Australian Bureau of Meteorology and CSIRO. Climate Variability, Extremes and Change in the Western Tropical Pacific: New Science and Updated Country Reports; Australian Bureau of Meteorology and CSIRO: Melbourne, Australia, 2014.

15. Durrant, T.; Greenslade, D.; Hemer, M.; Trenham, C. A Global Wave Hindcast focussed on the Central and South Pacific; CAWCR: Melbourne, Australia, 2014.

16. Ready, S.; Woodcock, F. The South Pacific and southeast Indian Ocean tropical cyclone season 1989-1990. Aust. Meteorol. Mag. 1992, 40, 111-121.

17. Gill, J.P. The South Pacific and southeast Indian Ocean tropical cyclone season 1991-1992. Aust. Meteorol. Mag. 1994, 43, 181-192.

18. Solomon, S.M. A Review of Coastal Processes and Analysis of Historical Coastal Change in the Vinicity of Apia, Western Samoa; South Pacific Applied Geoscience Commission: Suva, Fiji, 1994; Volume 208, p. 62.

19. Bureau of Meteorology, Samoa. Available online: http://www.bom.gov.au/pacific/samoa (accessed on 18 September 2015).

20. Rearic, D.M. Survey of Cyclone Ofa Damage to the Northern Coast of Upolu, Western Samoa; South Pacific Applied Geoscience Commission: Suva, Fiji, 1990; Volume 104, p. 36.

21. Carter, R. Design of the Seawall for Mulinu'u Point, Western Samoa; South Pacific Applied Geoscience Commission: Suva, Fiji, 1987; Volume 78, p. 30.

22. Holland, G. A Revised Hurricane Pressure-Wind Model. Mon. Weather Rev. 2008, 136, $3432-3445$. 
23. Booij, N.; Ris, R.C.; Holthuijsen, L.H. A third-generation wave model for coastal regions 1. Model description and validation. J. Geophys. Res. 1999, 104, 7649-7666.

24. Wu, J. Wind-stress coefficients over sea surface from breeze to hurricane. J. Geophys. Res. 1982, $87,9704$.

25. Huang, Y.; Weisberg, R.H.; Zheng, L.; Zijlema, M. Gulf of Mexico hurricane wave simulations using SWAN: Bulk formula-based drag coefficient sensitivity for Hurricane Ike. J. Geophys. Res. 2013, 118, 3916-3938.

26. Zijlema, M.; van Vledder, G.P.; Holthuijsen, L.H. Bottom friction and wind drag for wave models. Coast. Eng. 2012, 65, 19-26.

27. Janssen, P.A. E.M. Wave-Induced Stress and the Drag of Air Flow over Sea Waves. J. Phys. Oceanogr. 1989, 19, 745-754.

28. Saha, S.; Moorthi, S.; Wu, X.; Wang, J.; Nadiga, S.; Tripp, P.; Behringer, D.; Hou, Y.-T.; Chuang, H.; Iredell, M.; et al. The NCEP Climate Forecast System Version 2. J. Clim. 2014, 27, 2185-2208.

29. Lesser, G.R.; Roelvink, J.A.; van Kester, J.A. T.M.; Stelling, G.S. Development and validation of a three-dimensional morphological model. Coast. Eng. 2004, 51, 883-915.

30. Mulligan, R.P.; Walsh, J.P.; Wadman, H.M. Storm surge and surface waves in a shallow lagoonal estuary during the crossing of a hurricane. J. Waterw. Port. Coast. Ocean Eng. 2015, 141, A5014001.

31. Barnard, P.L.; van Ormondt, M.; Erikson, L.H.; Eshleman, J.; Hapke, C.; Ruggiero, P.; Adams, P.N.; Foxgrover, A.C. Development of the Coastal Storm Modeling System (CoSMoS) for predicting the impact of storms on high-energy, active-margin coasts. Nat. Hazards 2014, 74, 1095-1125.

32. Taebi, S.; Pattiaratchi, C. Hydrodynamic response of a fringing coral reef to a rise in mean sea level. Ocean Dyn. 2014, 64, 975-987.

33. Hoeke, R.K.; Storlazzi, C.D.; Ridd, P. V Drivers of circulation in a fringing coral reef embayment: A wave-flow coupled numerical modeling study of Hanalei Bay, Hawaii. Cont. Shelf Res. 2013, 58, 79-95.

34. Roelvink, J.A.; Walstra, D.-J. Ro keeping it simple bu using complex models. Adv. Hydro-Sci. Eng. 2004, 6, 1-11.

35. Lowe, R.J.; Falter, J.L.; Bandet, M.D.; Pawlak, G.; Atkinson, M.J.; Monismith, S.G.; Koseff, J.R. Spectral wave dissipation over a barrier reef. J. Geophys. Res. 2005, 110, 1-16.

36. Filipot, J.-F.; Cheung, K.F. Spectral wave modeling in fringing reef environments. Coast. Eng. Proc. 2012, 67, 67-79.

37. Madsen, O.; Poon, Y.; Graber, H. Spectral Wave Attenuation by Bottom Friction: Theory. Coast. Eng. Proc. 1988, 21, 492-504.

38. Raubennheimer, B.; Guza, R.T.; Elgar, S. Field observations of wave-driven setdown and setup. J. Geophys. Res. 2001, 106, 4629-4638.

39. Murakami, H. Tropical cyclones in reanalysis data sets. Geophys. Res. Lett. 2014, 41, 2133-2141.

40. Li, N.; Roeber, V.; Yamazaki, Y.; Heitmann, T.W.; Bai, Y.; Cheung, K.F. Integration of coastal inundation modeling from storm tides to individual waves. Ocean Model. 2014, 83, 26-42.

41. Hench, J. Episodic circulation and exchange in a wave-driven coral reef and lagoon system. Limnol. Oceanogr. 2008, 53, 2681-2694. 
42. Taebi, S.; Lowe, R.J.; Pattiaratchi, C.B.; Ivey, G.N.; Symonds, G.; Brinkman, R. Nearshore circulation in a tropical fringing reef system. J. Geophys. Res. 2011, 116, C02016.

43. Gourlay, M.R.; Colleter, G. Wave-generated flow on coral reefs-An analysis for two-dimensional horizontal reef-tops with steep faces. Coast. Eng. 2005, 52, 353-387.

44. Becker, J.; Merrifield, M.A.; Ford, M. Water level effects on breaking wave setup for Pacific Island fringing reefs. J. Geophys. Res. 2014, 119, 914-932.

45. Andersson, A.J.; Gledhill, D. Ocean acidification and coral reefs: Effects on breakdown, dissolution, and net ecosystem calcification. Ann. Rev. Mar. Sci. 2013, 5, 321-348.

46. Grady, A.E.; Moore, L.J.; Storlazzi, C.D.; Elias, E.; Reidenbach, M.A. The influence of sea level rise and changes in fringing reef morphology on gradients in alongshore sediment transport. Geophys. Res. Lett. 2013, 40, 3096-3101.

47. Buckley, M.; Lowe, R.; Hansen, J. Evaluation of nearshore wave models in steep reef environments. Ocean. Dyn. 2014, 64, 847-862.

48. Pomeroy, A.; Lowe, R. The dynamics of infragravity wave transformation over a fringing reef. J. Geophys. Res. 2012, 117, C11022.

49. Péquignet, A.-C.N.; Becker, J.M.; Merrifield, M.A. Energy transfer between wind waves and low-frequency oscillations on a fringing reef, Ipan, Guam. J. Geophys. Res. 2014, 119, 6709-6724.

(C) 2015 by the authors; licensee MDPI, Basel, Switzerland. This article is an open access article distributed under the terms and conditions of the Creative Commons Attribution license (http://creativecommons.org/licenses/by/4.0/). 